\title{
Erratum to: Kibdelosporangium phytohabitans sp. nov., a novel endophytic actinomycete isolated from oil-seed plant Jatropha curcas $\mathbf{L}$. containing 1-aminocyclopropane- 1-carboxylic acid deaminase
}

\author{
Ke Xing • Guang-Kai Bian · Sheng Qin • \\ Hans-Peter Klenk • Bo Yuan · Yue-Ji Zhang • \\ Wen-Jun Li $\cdot$ Ji-Hong Jiang
}

Published online: 27 October 2013

(C) Springer Science+Business Media Dordrecht 2013

Erratum to: Antonie van Leeuwenhoek (2012)

$$
\text { 101:433-441 }
$$

DOI 10.1007/s10482-011-9652-4

In the original published article, in page 436 , the value ' 0.092 ' in the sentence 'KLBMP $1111^{\mathrm{T}}$ exhibited high ACC deaminase activity $(0.092 \mu \mathrm{mol} \alpha$-ketobutyrate $\mathrm{mg}^{-1} \mathrm{~h}^{-1}$ )' is incorrect. The value ' 0.092 ' should be corrected to '33.294'.

The online version of the original article can be found under doi:10.1007/s10482-011-9652-4.

K. Xing · G.-K. Bian · S. Qin $(\bowtie) \cdot$ B. Yuan •

Y.-J. Zhang · J.-H. Jiang $(\bowtie)$

School of Life Science, The Key Laboratory of

Biotechnology for Medicinal Plant of Jiangsu Province,

Xuzhou Normal University, Xuzhou, Jiangsu 221116,

People's Republic of China

e-mail: shengqin@xznu.edu.cn

J.-H. Jiang

e-mail: jhjiang@xznu.edu.cn

H.-P. Klenk

DSMZ, Deutsche Sammlung von Mikroorganismen und Zellkulturen $\mathrm{GmbH}$, Inhoffenstraße 7b,

38124 Braunschweig, Germany

W.-J. Li

The Key Laboratory of Microbial Diversity in Southwest China, Ministry of Education and Laboratory for Conservation and Utilization of Bio-Resources, Yunnan Institute of Microbiology, Yunnan University,

Kunming 650091, People's Republic of China 